\title{
MACROMICETES DE LA COMARCA DEL BERGUEDA (BARCELONA)
}

\author{
J. M. LOSA QUINTANA; J. ANDRES RODRIGUEZ; R. CARBO NADAL \\ y O. GARCIA PRIETO*
}

RESUMEN:

Se da cuenta de ciento cuarenta y ocho especies de macromicetes recolectadas en el transcurso de una serie de excursiones de estudio, realizadas por los autores entre los años 1974 al 1977, por diversas localidades de la comarca del Berguedá (Barcelona).

Numerosas especies entre las indicadas fueron recolectadas en varias ocasiones, lo que permitió apreciar detalles de su comportamiento autoecológico.

En todos los casos hemos pretendido confirmar las determinaciones taxonómicas utilizando los medios reseñados en la Bibliografía. De cada especie se dispone de su ficha de estudio, con los detalles apreciados al realizar la determinanción, pese a ello no hemos considerado adecuado dar descripciones por no hacer más extenso el presente trabajo.

\section{SUMMARY:}

We remark the determinations of a hundred and forty eight species of Macromycets collected the year 1974 to the 1977, mainly in Berguedá district (Barcelona).

Most of them could be studied more than once. We tried to confirm the determinations using the last specialized works.

\section{INTRODUCCION}

La comarca del Berguedá, ha sido objeto de sucesivas excursiones de estudios micológicos entre los años 1974 al 1977, especialmente en la estación otoñal, época en la que pueden encontrarse en gran número, carpóforos de Macromicetes.

Por lo general se ha tomado como base de partida la ciudad de Berga, tendiendo a explorar todas las zonas de vegetación circundante, que son: hayedos, robledales húmedos, robledales submediterráneos y pinares. 
Otras excursiones a localidades relativamente cercanas a esta comarca, permiten añadir algunas especies que consideramos interesantes, sobre todo en bosques de abetos.

Pese a que en dichas excursiones la finalidad principal fue la recolección de cuerpos fructíferos para su posterior clasificación y determinación taxonómica, en especial de aquellas especies que nos eran relativamente conocidas, fuimos procurando relacionar las especies con las formaciones arbóreas, donde eran encontradas, ello nos ha permitido acompañar a las determinaciones taxonómicas, de algunos comentarios sobre características observadas en los lugares donde fueron recogidas, en particular relacionadas con aspectos autoecológicos.

Pese a que no ha sido nuestro propósito la realización de descripciones morfológicas de las especies estudiadas, se reseñan, en algunos casos, aspectos que consideramos importantes para la determinación, o confirmación nomenclatural.

El paisaje vegetal de esta comarca es característico por el predominio de bosques caducifolios y fuerte recubrimiento del suelo por la vegetación, tanto en el piso montano como a niveles superiores. Por encima de los $1.600 \mathrm{~m}$., se encuentran formaciones de pinos en las que predominan Pinus silvestris L., de donde proceden una parte de las especies estudiadas.

\section{METODOLOGIA}

En la mayoría de las especies que se indican se procuró confirmar las determinaciones, utilizando las obras especializadas mas recientes, asi para los Basidiomicetes se ha consultado el criterio nomenclatural de Moser, M.: «Basidiomyceten, (Agaricales)»: Kleine Kryptogamen Flora II,/b2; así como la revisión nomenclatural realizada por Lambinon en la obra de Darimont: "Recherches mycosociologiques dans les forets de Haute Belgique», 1973.

No hemos juzgado oportuno incluir descripciones ni detalles de las fichas realizadas, para evitar que el presente trabajo sea excesivamente largo.

En cada una de las excursiones de trabajo los ejemplares que fueron encontrados en ecotipos distintos fueron agrupadas en bolsas independientes, para poder dar con precisión los lugares donde fueron halladas.

Es a partir de las fichas de confirmación en el Laboratorio, como se ha podido realizar la siguiente lista de las especies estudiadas:

Agaricus augustus Fr.

Psalliota augusta (Fr.) Quelet; Berga 31-5-75

Especie saprófita que suele encontrarse en zonas aclaradas de bosques de Pinus.

Agaricus haemorrhoidarius Schulz.

Psalliota haemorrhoidaria (Schul) Fr.; Berga: Can Calvet 17-11-74.

Especie próxima a $A$. silvaticus Secret. aunque aparece mas bien en relación con bosques caducifolios mientras que $A$. silvaticus muestra preferencias por bosques de Pinos. 
Amanita phalloides (Fr.) Link

Berga: Cal Calvet: 17-11-74. Espinalbet: 12-11-75.

En claros de bosques de Pinus silvestris y matorral de degradación con Buxus sempervirens. Es común en otoño en matorrales y robledales con un carácter húmedo, aunque oscila mucho su aparición de unos años a otros.

\author{
Amanita pantherina (DC ex Fr.) Krombh. \\ Berga: 1-X-77 en bosques con Pinus silvestris L. \\ La Serreta: 18-9-75 en bosques de Pinus silvestris \\ Se muestra como especie de aparición precoz, busca claros de bosques sobre suelos de \\ carácter ligeramente ácido, en lugares sombrios y húmedos.
}

Amanita muscaria (L. ex Fr.) Hook

La Serreta: 18-9-75 en bosques de Pinus silvestris L.

Ejemplares de grandes tamaños que corresponden a la var. regalis Fr.; caracterizada por sus peculiaridades ecológicas, de tipo eurosiberiano.

Amanita rubescens (Pers. ex Fr.) S. F. Gray

Vall d'Aran (Lérida): 3-X-75 en bosques de Abies alba Miller

Destacan los ejemplares estudiados por su gran tamaño y el enrojecimiento de su carne en contacto con el aire.

Amanita vaginata (Bull. ex Fr.) Vitt.

Berga: 20-X-77 en bordes de zonas desforestadas del pinar de Pinus silvestris. Común en robledales caducifolios y pinares en el piso montano medio.

A propósito de esta especie sería interesante confiṛmar la presencia de la Amanita Mairei Foley (A. pseudovaginata Maire) frecuentemente confundida con la var. grisea, de A. vaginata y que fue señalada su presencia en el Sur de Francia (A. Parrot 1965 Bull. Centr. Recher. Etudes. sc. Biarritz 5 (3) p. 341).

\title{
Auriscalpium vulgare Karsten
}

Berga: 1-X-77 Pinar de Pinus uncinata Miller

Especie poco común, siempre sobre conos de Pinus, situados en lugares húmedos.

Boletus aereus Bull. ex Fr.

Berga: 19-X-74, en bosque de Pinus uncinata Miller.

Especies de carácter micorrícico, como la mayoría de las especies de este género.

Por sus caracteres microscópicos creemos pertenezca a esta especie, pese a que es difícil diferenciarla con B. pinicola Vitt.

\section{Boletus amarellus Qelet}

Berga: 2-X-77. Pinar de Pinus silvestris L.

Difiere de B. piperatus, por su tamaño mas reducido y sus esporas mas estrechas asi como por la presencia de cistidios hialinos, lisos. (Marchand, 1975 Doc. Myc. 21 (6) p. 40, indica una autoecología con preferencia de suelos ácidos en altitudes superiores a los $1.700 \mathrm{~m}$.). K. \& R. p. 41, lo consideran como una variedad de B. piperatus Fr. ex Bull. 
Boletus carpini Schulz

Martinet de Cerdaña: 10-X-75: en bosque de Pinus uncinata Miller.

Destaca la coloración de la carne pileica en rojo por el formol y el color azul verdoso por el sulfato de hierro.

Boletus castaneus Bull.

Berga: 3-X-75 en bosques de Pinus uncinata Miller.

Ampliamente representado en otoño, siendo numerosas las localidades en las que podemos citar su presencia.

Boletus erythoropus (Fr.) Krombh.

Vall d'Aneu (Lleida): 18-X-75, en bosques de Abies alba Miller.

En sus características macroscópicas destaca su estipe punteado de fibrillas granulosas y apretadas así como el color amarillento de la carne pileica.

Boletus luridus Schaef. ex Fr.

Vall d'Aneu: 18-X-75 Bosques de Abies alba Miller.

Boletus luteus L.

Berga: 1-X-77, bosques de Pinus silvestris L. y matorrales de sustitución con Buxus sempervirens $\mathrm{L}$. Especie fácilmente reconocible por los restos del velo general sobre el estipe. Especie micorrícica con diversas especies de Pinus.

\section{Boletus satanas Lenz}

Vall d'Aneu (Lleida): 18-X-77. Bosques de Abies alba Miller.

Destaca en los ejemplares estudiados la forma del estipe, corto, obeso y con un fino retículo parduzco. Especie micorrícica con diversas especies forestales.

Boletus subtomentosus L. ex Fr.

Xerocomus subtomentosus (L. ex Fr.) Quel.

Vall d'Aneu: 29-9-75: en bosques de Abies alba Miller

Boletus variegatus Swartz.

Berga: 1-X-77 en bosques de Pinus silvestris L.

Calocera viscosa Pers.

Vall d'Aneu (Lleida) 28-9-77 en bosques de Abies alba Miller.

Calodon nigrum (Fr.) Quel.

Sant Jaume: Berga: 13-X-74 en bosques de Pinus silvestris L. y matorrales de sustitución con Buxus sempervirens L.

Calvatia caelata Fr.

Berga: 15-5-75 en bosques de Pinus silvestris L. 
Confirmada la determinación por Les Gasteromycetes d'Espagne: Calonge \& Demoulin: 1975.

Cantherellus lutescens Pers. ex Fr.

Berga: 1-X-77; bosque de Pinus silvestris L. y matorrales de sustitución con Buxus sempervirens $\mathrm{L}$. Especie micorrícica.

Clavaria cinerea (Bull. ex Fr.) Schroet.

Berga: 1-X-77 en pinar de Pinus silvestris L.

Clavaria mairei Donk.

Berga: 29-9-75; bordes de caminos en el pinar de Pinus silvestris L.

Clavaria truncatula Quel.

Mont Cau, (Berga): 28-9-75, bosque mixto de Pinus silvestris L. y Pinus uncinata Miller, también en matorrales de sustitución.

Clavulina rugosa (Bull. ex Fr.) Schroet.

Berga: bosque de Pinus uncinata Miller 1-X-77

Clitocybe clavipes (Pers. ex Fr.) Kume.

Berga 1-X-77; bosque de Pinus uncinata Miller.

Por lo general sobre suelos ácidos o descalcificados.

Clitocybe dealbata (Sow. ex Fr.) Kumm.

Berga: 1-X-77; en prados húmedos.

El grupo de especies que abarca los Clitocybes de pileo blanco es complejo, y su diferenciación no siempre es fácil de realizar.

Clitocybe gibba (Pers. ex Fr.) Kumm.

C. infundibuliformis Schaef.

Berga: 18-X-75 en bosques de Pinus silvestris L.

Diversas variedades de esta especie han sido descritas; los ejemplares examinados pueden incluirse en la var. costata Fr.

Clitocybe odora (Bull. ex Fr.) Kumm.

Vall d'Aneu (Lleida), 22-9-76 en bosques de Abies albu Miller.

K. \& R. destacan la coloración verdosa de la cutícula piléica que observamos en los ejemplares recogidos, como un carácter que permite identificar esta especie.

Clitocybe suaveolens (Schum. ex Fr.) Kumm.

Berga: 1-X-77, en claros del bosque de Pinus silvestris L.

Especie saprófita que tiene en fresco un suave olor anisado. 
Collybia butiracea (Bull. ex Fr.) Quel.

Berga: 1-X-77 en claros del Pinar de Pinus silvestris L.

Reconocible por su mamelón pileico central y su tacto untuoso en fresco.

Collybia maculata (Albert. \& Schw.) Quel.

Vall d'Aran (Lleida) 20-9-77 en bosques de Abies alba Miller.

Berga: 1-X-77, en bosques de Pinus silvestris L. También en matorrales de sustitución con Buxus sempervirens L.

Coprinus comatus (Müll. ex Fr.) S.F, Gray

Berga: 9-5-75 en zonas aclaradas y algo nitrófilas del pinar de Pinus silvestris L.

Especie saprófita de frecuente aparición a principios de otoño, si bien fue encontrada también en primavera. Prefiere zonas pisadas y algo nitrófilas con elevada humedad.

Cortinarius caerulescens Schaef. ex Fr.

Berga: 2-X-77 en bosques de Pinus silvestris L.

Destacan las laminillas de intenso color azul violáceo, que también se observa en la cutícula pileica y en el estipe.

Cortinarius cinnamomeus (L.) Fr.

Berga: 2-X-77 en bosque de Pinus silvestris L.

K. \& R. indican que bajo esta denominación Fries reunió diversos tipos que difieren por el color de las laminillas.

Cortinarius saturninus Fr. s.s. R. Henry

Berga: 1-X-77 en bosques de Pinus silvestris L.

Parece comportarse como especie micorrícica con diversas especies de Pinus.

Crepidotus variabilis Pat.

Berga: 1-X-77, en bosque de Pinus silvestris L.

La mayoría de las especies de este género son lignícolas y epífitas, los carpóforos de tamaños siempre reducidos suelen aparecer sobre tocones.

Cystoderma amianthinum (Scop. ex Fr.) Fayod

Berga: 1-X-77 en bosques de Pinus silvestris L. y matorrales de sustitución con Buxus sempervirens $\mathrm{L}$.

Aparece por lo general en zonas aclaradas o bordes de pastizales, aunque se comporta como especie micorrícica.

Cystoderma carcharias (Pers.) Fayod.

Berga: 1-X-77 en bosques de Pinus silvestris L. y Pinus uncinata Miller.

Por lo general micorrícica con diversas especies de Pinus.

Cystoderma cinnabarinum (Alb. et Schw. ex Fr.) Fayod.

Berga: 2-X-77 en el pinar de Pinus silvestris L. 
Destacan como caracteres microscópicos el revestimiento cuticular piléico en forma de escamas fibrillosas y la abundancia de cistidios marginales.

Cystoderma granulosum (Batsch.) Fayod.

Berga: 19-X-75 en el bosque de Pinus silvestris L.

Cystoderma granulosum (Batsch.) Fayod.

Berga: $19-\mathrm{X}-75$ en el bosque de Pinus silvestris L.

No se observaron cistidios marginales, ni faciales.

Fistulina hepatica Schaeff ex Fr.

Vall d'Aneu (Lleida) 28-9-77, sobre tocones de Abies alba Miller.

Ganoderma lucidum (Lyss. ex Fr.) Karst.

Berga: 29-9-75. Sobre restos lignificados de tocones de Pinus silvestris L.

Geastrum rufescens (Pers.) Fr.

Berga: 1-X-77 en bosques de Pinus silvestris L. y matorrales de sustitución con Buxus sempervirens $\mathrm{L}$.

(Boiffard, J. 1976, publica en Doc. Myc. 6 (24) p. 23, un estudio monográfico del género Geastrum, en él separa esta especie de, G. sessile (Sow.) Pouzar, $=$ G. rufescens Pers p.p. por el examen de las esporas).

Gloeophyllum saepiarium (F.) Karst.

Trametes saepiaria (Wulf. ex Fr.) Pilat

Berga: 1-X-77 sobre restos lignificados de Pinus uncinata Miller.

Cuerpo fructífero muy coriáceo de color ocráceo.

Gomphidius glutinosus Scop.

Berga: 1-X-77, en bosques de Pinus silvestris L. y matorrales de sustitución.

Especie micorrícica con diversas especies de coníferas.

\section{Gomphydius viscidus $\mathrm{L}$.}

Especie frecuentes y de fácil identificación por su estipe

Berga: 1-X-77; en el pinar de Pinus silvestris L.

Gymmopilus penetrans (Fr.) Murr.

Flammula penetrans $\mathrm{Fr}$.

Berga: 1-X-77; sobre piñas en el suelo del pinar de Pinus uncinata Miller.

No se observó la presencia de velo marginal, por lo que pude incluirse en la forma típica.

Guepina helvelloides DC.

Gyrocephalus rufus (Jacq.) Bref. 
Berga: 1-X-77, en lugares muy húmedos del pinar de Pinus silvestris L.

Cuerpo fructífero gelatinoso y superficie fértil exterior.

Helvella crispa Scop. ex Fr.

Berga: 1-X-77 en claros del bosque de Pinus silvestris L.

Helvella lacunosa Afz. ex Fr.

Vall d'Aneu (Lleida) en bosques de Abies alba Miller.

Helvella sulcata Afz. ex Fr.

Berga: 28-9-75, en claros del bosque de Pinus silvestris L.

Hericium erinaceus (Fr.) Quel.

Montmajor, Berga: 17-9-75 en el bosque de Quercus pubescens Willd.

Los ejemplares de gran tamaño, uno de ellos alcanzó los dos kgr. de peso.

Hydnellum ferrugineum (Fr. ex Fr.) Karst.

Calodon ferrugineum (Fr.) Pat.

Vall d'Aran (Lleida), 20-9-77, en bosques de Abies alba Miller.

Hydnum repandum L. ex Fr.

Berga: 28-9-77, en bosques de Pinus silvestris L.

Hygrocybe coccinea (Schaef. ex Fr.) Karst.

Hygrophorus coccineus Scop.

Berga: 1-X-77 en claros del bosque de Pinus silvestris L.

Siempre en zonas con elevada humedad.

Hygrocybe conica (Scop. ex Fr.) Karst.

Hygrophorus conicus Fr.

Berga: 1-X-77, bosque de Pinus silvestris L.

En pastizales húmedos o próximos a cursos de agua.

Hygrocybe chlorophana (Fr.) Karst.

Hygrophorus chlorophanus $\mathrm{Fr}$;

Berga: 1-X-77 en el pinar de Pinus silvestris L.

Destaca el color amarillo del estipe, igual al del pileo y muy brillante.

Hygrocybe punicea (Fr.) Karts.

Hygrophorus puniceus $\mathrm{Fr}$.

Berga: 1-X-77, en claros del pinar de Pinus silvestris L.

Hygrocybe psittacina (Schaeff. ex Fr.) Karst.

Hygrophorus psittacinus Fr.

Siempre en lugares de elevada humedad. 


\section{Hygrophorus agathosmus Fr.}

Berga: 3-X-75, en claros del bosque de Pinus silvestris L.

Especie micorrícica del grupo Limacium, que se reconoce por el suave olor de sus carpóforos y sus laminillas decurrentes.

Hygrophorus chrysodon (Batsch. ex Fr.) Fr.

Berga: 1-X-77, en claros del bosque de Pinus silvestris L.

Recuerda por sus capóforos al $H$. eburneus, aunque suele distinguirse bien por los restos sobre el estipe de un velo que suele tener tonalidades amarillentas.

Hygrophorus dichrous Kuhn. \& Romag.

Berga: 2-10-77, en claros del bosque de Pinus silvestris L.

Destaca su mamelón pileico de color oliváceo y el estipe de color blanco.

Hygrophorus eburneus (Bull. ex Fr.) Fr.

Berga: 17-8-74, en claros del bosque de Pinus silvestris L.

Especie que presenta una variada diversidad de formas, que algunos autores describen como variedades.

\section{Hygrophorus gliocyclus Fr.}

Berga: 1-X-77, en claros del pinar de Pinus silvestris L.

Especie micorrícica con diversas coníferas; se destaca su carpóforo por la viscosidad de su cutícula pileica.

Hygrophorus hipothejus (Fr.) Fr.

Berga: 4-X-75; en bosques de Pinus uncinata Mill.

Especie que presenta gran variabilidad por la tonalidad de su cutícula pileica, los ejemplares estudiados pueden incluirse en la var. aureus (Arrh. ap. Fr.) Imler.

\section{Hygrophorus pratensis (Pers.) Kummer}

Berga: 1-X-77 en bosques de Pinus uncinata Mill.

Se sitúa taxonómicamente cerca de $H$. nemoreus Lasch ex Fr. y ambas especies tienen carácter micorrícico, si bien en ésta los carpóforos suelen aparecer en claros del bosque.

\section{Hygrophorus pudorinus (Fr.) Fr.}

Valle de Arán (Lérida): 20-9-77, en bosques de Abies alba Miller.

Los carpóforos son identificables por su coloración y porte típiços.

Hygrophorus russula (Schaef. ex Fr.) Quel.

Berga: 21-X-74, en bosque mixto con Quercus ilex $\mathrm{L}$.

Especie que prefiere aparecer en encinares, con los que tiene micorrizas, es fácilmente reconocible por su forma y coloración pileíca.

Hypomyces lateritius Fr.

Micromicete que se desarrolla sobre carpóforos de diversas especies de hongos.

Fue estudiado sobre Lactarius deliciosus L. 
Hypholoma fasciculare (Huds. ex Fr.) Kumm.

Berga: 2-X-77, sobre tocones de Pinus silvestris L.

Inocybe calamistrata Fr.

Berga: 1-X-77, en el borde de senderos en el pinar de Pinus silvestris L.

Especie terrícola, subhigrófila, que aparece en grupos en el mantillo del pinar.

Inocybe fastigiata (Schaeff. ex Fr.) Quel.

Berga: 2-X-77, en bosques de Pinus silvestris L.

Especie polimorfa, aunque las variedades no siempre son fáciles de separar.

Inocybe geophylla (Sow. ex Fr.) Kumm.

Berga: 1-X-77, en zonas sombrías y húmedas del pinar de Pinus silvestris L.

Lactarius mitissimus (Fr.) Fr.

Valle de Arán (Lérida): 20-9-77 en bosques de Abies alba Miller

Frecuente en bosques de coníferas en el piso montano, con los que se comporta como especie micorrícica.

Lactarius sanguifluus Paulet.

Berga: 3-X-76: en bosques de Pinus silvestris L.; 2-X-77 y en matorrales de sustitución con Buxus sempervirens L.

Lactarius scrobiculatus (Scop. ex Fr.) Fr.

Vall d'Arán (Lérida) 20-9-77, en bosques con Abies alba Mill.

Lactarius subdulcis (Bull. ex Fr.) S.F. Gray

Berga: 1-X-77; en bosques mixtos con Quercus pubescens. Willd.

Especie micorrícica con diversas especies, que también aparece en hayedos.

Lactarius torminosus (Schaef. ex Fr.) S.F. Gray

Vall d'Aran (Lleida): 18-X-75, en bosquetes de Betula sp.

Fácil de reconocer por los largos pelos que tienen los carpóforos en el borde de las laminillas, las formas más pálidas en la coloración cuticular se consideran como var. cilicioides Konr. \& Maub.

Lactarius zonarius (Bull. ex St. Amans) Fr.

Espinalbet, Berga: 20-X-75, en bosques de Pinus silvestris L.

Dentro de esta especie han sido consideradas una serie de formas, de las cuales la var. zonarioides Künh. \& Romag., es propia de bosques de coníferas en el piso montano y a la cual pertenecen los ejemplares estudiados.

Lepiota procera (Scop. ex Fr.) S.F. Gray

Berga: 10-X-74, en claros del bosque de Pinus silvestris L.

También en bosques mixtos y matorrales de sustitución de Buxo-Quercetum pubescentis; sobre suelos ligeramente ácidos. 
Lepiota rhacodes (Vitt.) Quel.

Berga: 18-X-74, en bordes de caminos y claros del bosque mixto, con Pinus silvestris L. Destacan para su diferenciación el estipe blanco sin escamas y la coloración rosa que adquiere la carne pileica al cortarla.

Lepista luscina (Fr. ex Fr.) Singer

Rhodopaxillus panaeolus (Fr.) Maire

Can Calvet, Berga en el matorral de sustitución del bosque mixto, predominando Buxus sempervirens $\mathrm{L}$.

Lepista nuda (Bull. ex Fr.) Cooke

Rhodopaxilus nudus (Bull.) Maire

Can Calvet, Berga 17-9-74, en zonas umbrías y húmedas del pastizal.

Especie saprófita que aparece en círculos.

Leucoporus arcularius (Bull.) Quel.

Berga, 1-X-77, en el bosque mixto con Pinus silvestris L.

Incluible en el grupo de Leucoporus brumalis (Pers. ex.) Quel, separándose por los poros alveolares oblongos y mayores.

Lycogala epidendron Buxb.

Berga, 1-X-77, sobre leños en vías de degradación.

Lycoperdon excipuliforme (Scop. ex Pers.) em. Perdeck.

La Vall d'Aneu (Lleida) 20-9-75, en el bosque con Abies alba Mill.

Del grupo Calvatia, fácil de diferenciar por su porte y por que al cortar la gleba pasa de color blanco a ligeramente oliváceo.

Lycoperdon molle Pers.

Berga: 2-X-77, en bordes de caminos en el bosque mixto con Pinus silvestris L.

Lycoperdon perlatum Pers. ex Pers.

L. gemmatum Batsch.

Berga: 10-X-74, en claros del matorral de sustitución del bosque mixto.

Lycoperdon pyriforme Schaeff ex Pers.

Berga: 1-X-77 en el bosque mixto con Pinus silvestris L.

De comportamiento micorrícico con esta conífera.

Lyophyllum inmundum (Berk.) Kühn.

Tricholoma fumosum Ricken

Berga: 1-X-77, en el bosque mixto con Pinus silvestris L.

Marasmius collinus (Sow ex Fr.) Singer

Berga: 1-X-77 en el pastizal dentro del pinar.

Especie próxima a $M$. oreades Bolt. ex Fr.; anteriormente había sido incluída dentro del género Collybia, por su habitat en pastizales húmedos. 
Melanoleuca cognata (Fr.) Konr. \& Maub.

Berga: 1-X-77, en el bosque de Pinus uncinata Miller

Determinada por la coloración ocrácea-rosada de las laminillas y por los cistidios fusiformes de las mismas.

Melanoleuca grammopodia (Bull.) Pat.

Berga: 2-X-77, en el pinar con Pinus silvestris L. formando parte del pastizal donde llega a formar a modo de círculos.

Mycena acicula (Shaef. ex Fr.) Kumm.

Berga: 1-X-77, en claros de bosque mixto con Pinus silvestris L.

Recogidos los carpóforos en lugares sombríos y húmedos, destaca su píleo de color anaranjado en el centro, y la presencia de cistidios en las laminillas.

Mycena pelianthina (Fr.) Quel.

Berga: 1-X-77, en lugares húmedos del bosque mixto.

Ejemplares con el sombrerillo de color violáceo y laminillas con el borde purpúreo, provistas de cistidios.

Mycena pura (Pers. ex Fr.) Kumm.

Berga: 1-X-77, en lugares húmedos del bosque mixto.

Especie muy frecuente y variable en su forma. Micorrícica.

Nectria cinnabarina Fr;

En leños en vías de destrucción; bosque mixto: Berga 2-X-77.

Otidea alutacea (Pers. ex Pers.) Mass.

Berga: 1-X-77, en el bosque con Pinus uncinata Mill.

Paneolus acuminatus Fr.

Berga: 1-X-77, en el bosque mixto.

Ejemplares recogidos en el pastizal sobre humus, presentando un pileo cónico y un estipe delgado y largo.

Panus conchatus Fr.

Can Calvet, Berga sobre restos de madera 29-9.77.

Destacan las laminillas de color ocráceo, decurrentes y con la arista provista de cistidios

Peziza badia Fr.

Vallcebre, Berga: 31-5-75, en suelo arenoso dentro del bosque mixto.

Phaeolepiota aurea (Matt.) Gillet.

Cystoderma aureum (Fr. ex Matt.) Kuhn. \& Romag.

Berga: 4-X-75, en zonas aclaradas del pinar de Pinus uncinata Mill.

Especie de difícil encuadre taxonómico, para la cual fue creada este género. 
Phalus impudicus L. ex Pers.

Montseny: en bordes de caminos del bosque de repoblación con abetos.

Recogidos ejemplares en diversos estadios de desarrollo.

Pholiota flammans (Fr.) Quel.

Berga: sobre tocones de Pinus, 2-X-77.

Destaca la presencia de mechas en la cutícula pileica, que son escuarrosas en el pie.

Pholiota squarrosa (Pers. ex Fr.) Kümm.

Vall d'Aran (Lleida) 20-9-77, sobre tocones de abetos.

Pleurotus ostreatus (Jacq. ex Fr.) Kümm

Berga: 10-12-74, sobre restos lignificados y maderas en vías de destrucción.

Los ejemplares presentan una esporada ligeramente rosada.

Pluteus cervinus (Schaeff. ex Fr.) Kümm.

Berga: sobre tocones en el pinar de Pinus silvestris L. 1-X-77

Han sido descritos diversas formas, en función a variaciones en los cistidios y pelos marginales, muy abundantes en las aristas de las laminillas.

Pluteus murinus Bres.

Berga: pinar de Pinus silvestris L. 1-X-77

Incluible en el grupo del $P$. villosus.

Pseudoclitocybe cyathiformis (Bull. ex Fr.) Sing.

Clitocybe cyathiformis Bull.

Omphalia cyathiformis Kühn. \& Romag.

Berga: Bosque de Pinus silvestris L. 4-X-1975

Especie que suele aparecer tardiamente en pastizales de suelos poco ácidos.

Laminillas netamente decurrentes y con una tonalidad rosada en la desecación.

Entoloma sericeum (Bull. ex Merat) Quel.

Rhodophyllus sericeus (Fr.) Quel.

Berga: 1-X-77, en el bosque de Pinus silvestris L.

Russula adulterina Fr. ss. Melz. \& Zv.

Berga: 2-X-77, en el bosque de Pinus silvestris L.,

Cutícula pileica de color pardo y carne de sabor acre. Esporada amarilla, esporas grandes y ornamentales. Cistidios voluminosos.

\section{Russula amethystina Quel.}

Berga: 2-X-77, en el bosque mixto de Quercus pubescens y Pinus silvestris.

Confundible por su aspecto con la $R$. turci, al tener la cutícula de tono violáceo. Especie micorrícida con diversos coníferas. 
Russula aurata With ex Fr.

Vall d'Aneu (Lleida) 15-IX-1975, en el bosque de Abies alba Mill.

Russula badia Quel.

Berga 2-X-77; en el bosque mixto con Pinus silvestris L.

Es quizá la especie de carne con sabor mas acre. Micorrícica con diversas coníferas.

Russula caerulea (Pers) Fr. ss. Cooke

R. amoenata Britz

Berga: 1-X-77, en claros del pinar de Pinus uncinata Mill.

Especie calcífuga de pinares del piso montano superior.

Destaca el mamelón pileico en una depresión central. Carne de sabor amargo.

Russula cyanoxantha (Schaeff. ex Schwein) Fr.

Vall d'Aneu Lleida 24-9-1977 en el bosque de Abies alba Mill.

Russula delica Fr.

Espinalbet, Berga: 31-8-1975, en el bosque mixto con Pinus silvestris L.

Russula emetica (Schaef. ex Fr. Pers. ex S.F. Gray, var. luteolactata Rea

Berga: 2-X-77, en zonas de pinares de Pinus silvestris L.

Considerada como especie independiente por Marchand (1977) que fue base de su determinación inicial. Aparece en lindes del bosque en contacto con la robleda y sobre suelos un poco descarbonatados.

\section{Russula integra $\mathrm{L}$.}

Berga: 1-X-77, en el pinar del piso montano superior con Pinus uncinata Mill.

\section{Russula mustelina Fr.}

$R$. elephantina Fr. ss. Singer

Berga: 1-X-77, en el pinar de Pinus uncinata Mill.

Destaca su reacción de la carne con el sulfato ferroso, tomando una coloración parda. Esporada de color crema, como las laminillas.

Russula nitida (Pers. ex Fr.) Fr.

Espinalbet, Berga. 31-X-1975, en el bosque mixto del piso montano medio, y con Betulas, busca sustratos húmedos. Marchand destaca su carácter micorrícico con los abedules.

Russula sanguinea Bull. ex Fr.

Espinalbet, Berga: 31-X-1975, en estaciones húmedas del pinar montano, sin llegar a contactar con el piso del Pinus uncinata. Mill.

Russula torulosa Bres. var. fuscorubra (Bres.) Blm.

Berga: $1-\mathrm{X}-77$, en bosque mixto, con Pinus y en suelos arenosos. 
Fue determinada y confirmada por Marchand (1977), comprobando sus caracteres en especial el sabor acre de su carne.

Russula vesca Fr.

Berga: 1-X-77; en el bosque mixto con Pinus silvestris L. y suelos ácidos

Russula xerampelina (Schaeff. ex Secret.) Fr.

Berga: 1-X-77, en el pinar de repoblación, si bien alcanza el piso del Pinus uncinata Mill. Prefiere suelos ácidos.

Sarcodon imbricatus (L. ex Fr.) Karst.

Berga: 9-9-77, frecuente en zonas aclaradas y degradadas del pinar.

Spathularia flavida Pers ex Fr. var. alpestris Rehm.

Berga: $21-9-75$, relacionada con los pinares del piso subalpino;

Stereum hirsutum (Willd. ex Fr.) S.F. Gray

Espinalbet, Berga: 8-3-76; relacionado con tocones de diversas especies arbóreas, fácil de renococer por la cutícula exterior lanoso-zonada con diversas tonalidades.

Stropharia aeruginosa (Fr.) Quel.

Geophila (Stropharia) aeruginosa (Curt. ex Fr.) Quel.

Berga: 18-X-75, en estaciones donde se descompone materia orgánica de origen animal.

Stropharia semiglobata (Batsch ex Fr.) Quel.

Berga: 1-X-77, en claros del pinar de Pinus silvestris L., sobre estiércol.

Trametes betulina (L. ex Fr.) Fr.

Lenzites betulina (L. ex Fr.) Pilat.

Espinalbet, Berga: 8-3-76, saprófita sobre tocones.

Trametes gibbosa (Pers. ex Fr.) Fr.

Berga: 28-9-75, sobre tocones de Fagus silvatica L. en los que provoca una podredumbre muy activa.

Coriolus versicolor (L. ex Fr.) Quel.

Trametes versicolor (L. ex Fr.) Pilat.

Vall d'Aneu (Lleida) 24-9-77, en tocones de Abies alba.

Tremella mesenterica Retz. ex Hook.

Berga: 18-X-77, sobre madera muerta a la que contribuye a descomponer. 
Tricholoma ustaloides Romag.

Berga: 1-X-77, en el bosque mixto con Pinus silvestris $\mathrm{L}$.

Tricholoma album (Schaeff ex Fr.) Quel.

Berga: 29-9-75, en el hayedo; destaca el sabor acre de su carne.

Tricholoma equestre (L.) Quel.

Berga: 10-X-74; en el bosque mixto, aunque es más común en pinares sobre suelos ácidos.

Tricholoma georgii (Clus.) Quel.

En prados y zonas aclaradas del bosque mixto.

Tricholoma pardinum Quel.

Vall d'Aran (Lleida) 10-9-77, en el bosque de Abies alba Mill.

Tricholoma portentosum (Fr.) Quel.

Berga: 4-X-75; en el bosque mixto con Pinus silvestris.

Tricholoma rutilans (Schaeff. ex Fr.) Sing.

Can Calvet, Berga: 29-9-75; sobre tocones de Pinus silvestris L.

Incluible en el grupo de Tricholomas lignícolas para los que Pilat propuso la denominación genérica de Tricholomopsis.

Tricholoma sulphureum (Bull. ex Fr.) Kumm.

Berga: 1-X-77; común en claros del bosque mixto con Pinus silvestris L.

Tricholoma terreum (Schaeff. ex Fr.) Kumm.

Espinalbet, Berga: 17-9-74; Frecuente en claros del bosque y en bordes de matorrales de sustitución del mismo, con Pinus silvestris L.

Leucopaxilus tricolor (Peck) Kühner

Can Rebeu, Berga: 26-X-75, en claros del bosque mixto con Pinus silvestris L., sobre suelos ácidos.

Volvaria parvula Weinm

Can Calvet, Berga: 28-X-76

Volvariella volvacea (Bull. ex Fr.) Sing.

La Clusa, Berga: 28-8-76

Xilaria polymorpha (Fr.) Grev.

Berga: 2-X-77, sobre tocones de Pinus silvestris L. 
Las abreviaturas empleadas corresponden a las siguientes obras:

K. \& R.: Künher, R. \& Romagnesi, H.: Flore analytique des Champignons superieurs; Masson et Cie Paris 1974.

M. \& B.: Malençon, G. \& Bertault, R.: Flore des Champignons superieurs du Maroc. I; Fd. des Sciences Rabat, 1970.

Moser.: Moser, M.: Basidiomyceten II (Agaricales) in Kleine Kryptogamen flora, H. Gams; II / b2, Gustav Fischer Verlag Stuttgart 1967.

S. M. de F.: Bull. Societé Mycologique de France: colección del n. ${ }^{0} 1$ al 74.

Doc. Myc.: Documents Mycologiques. Grupe de Mycologie fondamentale et appliquée, 59 Lille-France; núms. 1 al 27.

\section{Agradecimientos}

Este trabajo no hubiera podido ser realizado sin la decisiva colaboración de la Sociedad Micológica de Berga (Barcelona) y, especialmente de sus miembros: Ramón Ferrer Gasol, Juan Luis Sala Vilar y Domenech Cosp Sitges, que pusieron a nuestra disposición gran cantidad de material de todo tipo y que además, subvencionaron las campañas de recogida de muestras.

\section{BIBLIOGRAFIA}

CODINA, J. \& FONT QUER, P.: 1930. Introducció a l'estudi dels macromicets de Catalunya. Cavanillesia, 3. Barcelona.

HEIM, R.: 1934. Fungi Iberici. Observations sur la Flore Mycologique Catalane. Treb. Museu Ciencias Nat. de Barcelona 16: 1-146.

KONRAD, P. et MAUBLANC, A.: 1924/35. Icones selectae fungorum. Paris.

LANGE, J. E.: 1935/40.: Flora Agaricina Danica. Copenhague.

LOSA QUINTANA, J. M.: 1960. Contribución al estudio de los Inocybes españoles. C.S.I.C.: Cavanilles Sección Barcelona.

MAIRE, R.: 1933. Fungi Catalaunici. Contributions a l'etude de la Flore Mycologique de Catalogne. Treb. Museu Ciencias Nat. de Barcelona, 15: 1-122.

- 1937. Fungi Catalaunici, Series altera. Public. Inst. Botanic; 3, 4. Barcelona.

MALENÇON, G. \& BERTAULT, R.: 1971. Champignons de la Peninsule Iberique. Acta Phytotax. Barcinonensia, 8: 1-98.

- 1972. Champignons de la Peninsule Iberique II. Acta Phytotax. Barcinonensia, 9: 1-64.

- 1976 Champignons de la Peninsule Iberique, V. acta Pbytotax. Barcinonensia 19:1-68.

SCHAEFFER, J:: 1800. Fungorum qui in Bavaria et circa Ratisbonam nascuntur icones. Erlangae. 